\title{
A IMPORTÂNCIA DA
}

ASSISTÊNCIA DE ENFERMAGEM

NA ATENÇÃO BÁSICA À SAÚDE

\section{Volume 1}

ORGANIZADORES:

Andressa Prates Sá/ Dayane Araújo Rocha Keilla Silva Santos/Ingrid Isabel de Andrade Nhayeno Cordeiro Dantas/ Vinícius Duarte Silva Fernanda Santos Landim/ Francielle Araujo Bispo Weidny Eduardo de Sousa Silva/ Cecília Rodrigues Lima Anna Christina dos Reis Santos/ Thais Pereira Silva 


\section{A IMPORTÂNCIA DA}

ASSISTÊNCIA DE ENFERMAGEM

NA ATENÇÃO BÁSICA À SAÚDE

\section{Volume 1}

ORGANIZADORES:

Andressa Prates Sá/ Dayane Araújo Rocha Keilla Silva Santos/Ingrid Isabel de Andrade Nhayeno Cordeiro Dantas/Vinícius Duarte Silva Fernanda Santos Landim/ Francielle Araujo Bispo Weidny Eduardo de Sousa Silva/ Cecília Rodrigues Lima Anna Christina dos Reis Santos/ Thais Pereira Silva 
A IMPORTÂNCIA DA ASSISTÊNCIA DE ENFERMAGEM NA ATENÇÃO BÁSICA À SAÚDE

Volume: 1

$1^{\text {a }}$ Edição

TRIUNFO - PE 


\section{Editor-Chefe}

Me. Daniel Luís Viana Cruz

\section{Organizadores}

Andressa Prates Sá

Dayane Araújo Rocha

Keilla Silva Santos

Ingrid Isabel de Andrade

Nhayeno Cordeiro Dantas

Vinícius Duarte Silva

Fernanda Santos Landim

Francielle Araujo Bispo

Weidny Eduardo de Sousa Silva

Cecília Rodrigues Lima

Anna Christina dos Reis Santos

Thais Pereira Silva

\section{Conselho Editorial}

Dr. Cássio Brancaleone

Dr. Marcelo Luiz Bezerra da Silva

Dra. Pauliana Valéria Machado Galvão

Dr. Plínio Pereira Gomes Júnior

Dr. Walter Santos Evangelista Júnior

Dr. Wendel José Teles Pontes

Editores De Área - Ciências Da Saúde

Dra. Camyla Rocha de Carvalho Guedine

Dra. Cristieli Sérgio de Menezes Oliveira

Dr. Leandro dos Santos

Dr. Hugo Barbosa do Nascimento

Dr. Marcio Luiz Lima Taga 
Dra. Pauliana Valéria Machado Galvão

\section{Assistente Editorial}

Thialla Larangeira Amorim

\section{Imagem de Capa}

Freepik

\section{Edição de Arte}

Vileide Vitória Larangeira Amorim

\section{Revisão}

Os autores

\section{(2) $\odot \Theta \Theta$}

Este trabalho está licenciado com uma Licença Creative Commons - Atribuição-NãoComercialSemDerivações 4.0 Internacional.

O conteúdo abordado nos artigos, seus dados em sua forma, correção e confiabilidade são de responsabilidade exclusiva dos autores. 


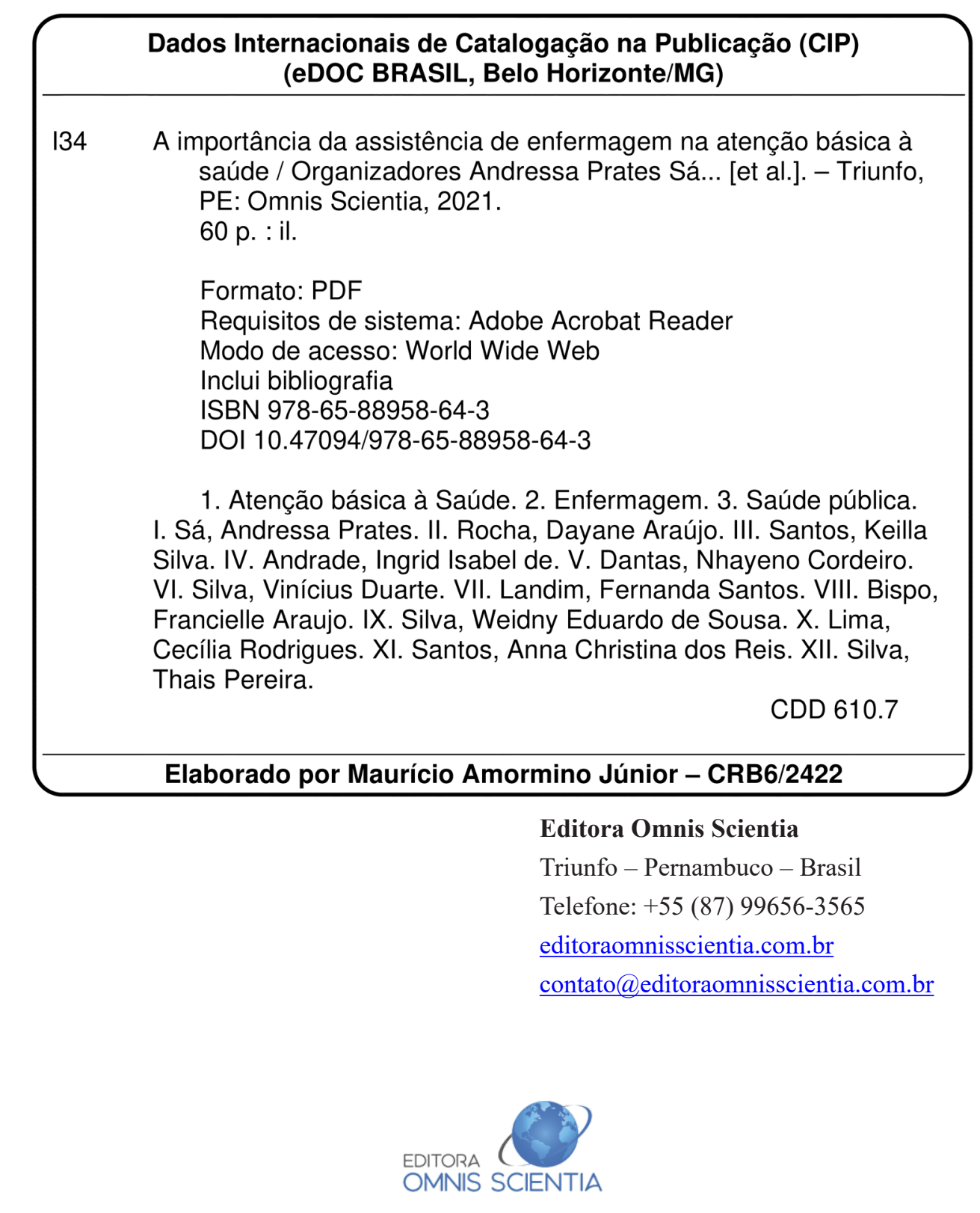




\section{PREFÁCIO}

O enfermeiro possui um papel fundamental dentro da atenção primária, pois nesse contexto esse profissional deve identificaros problemas desaúdee fatores de risco da população, monitoraras evoluções clínicas dos pacientes, participar e realizar ações voltadas para educação em saúde, realização e acompanhamento de tratamentos/reabilitação e a sistematização do cuidado dentro da atenção básica em todas as fases da vida humana, desde a gestação até a morte, dentro do cuidado integral do paciente, o atendendo no seu entorno biopsicossocial, assim concretizando várias das diretrizes do sistema único de saúde.

Este livro trás em seus capítulos estudos que evidenciam a necessidade e importância do trabalho desses profissionais da enfermagem dentro do sistema de saúde brasileiro, mostrando sua atuação dentro das diversas áreas, como na saúde da mulher, doenças crônicas e infectocontagiosas. 


\section{SUMÁRIO}

CAPÍTULO 1 . .12

A IMPORTÂNCIA DA ASSISTÊNCIA DE ENFERMAGEM AOS PACIENTES PORTADORES DE HANSENÍASE

Andressa Prates Sá

Dayane Araújo Rocha

Keilla Silva Santos

Ingrid Isabel de Andrade

Nhayeno Cordeiro Dantas

Vinícius Duarte Silva

Fernanda Santos Landim

Francielle Araujo Bispo

Weidny Eduardo de Sousa Silva

Cecília Rodrigues Lima

Aldair Almeida Batista

Thais Pereira Silva

DOI: 10.47094/978-65-88958-64-3/12-19

\section{CAPÍtULO 2.}

A RELEVÂNCIA DA EQUIPE DE ENFERMAGEM NA ASSISTÊNCIA DO CÂNCER DE COLO DO ÚTERO

Andressa Prates Sá

Dayane Araújo Rocha

Keilla Silva Santos

Ingrid Isabel de Andrade

Nhayeno Cordeiro Dantas

Vinícius Duarte Silva 
Fernanda Santos Landim

Francielle Araujo Bispo

Weidny Eduardo de Sousa Silva

Cecília Rodrigues Lima

Anna Christina dos Reis Santos

Thais Pereira Silva

DOI: 10.47094/978-65-88958-64-3/20-27

CAPÍTULO 3

A IMPORTÂNCIA DA ASSISTÊNCIA DA EQUIPE DE ENFERMAGEM AOS PACIENTES COM HIPERTENSÃO ARTERIAL SISTÊMICA

Andressa Prates Sá

Dayane Araújo Rocha

Keilla Silva Santos

Ingrid Isabel de Andrade

Nhayeno Cordeiro Dantas

Vinícius Duarte Silva

Fernanda Santos Landim

Francielle Araujo Bispo

Weidny Eduardo de Sousa Silva

Cecília Rodrigues Lima

Anna Christina dos Reis Santos

Thais Pereira Silva

DOI: 10.47094/978-65-88958-64-3/28-36 
ASSISTÊNCIA DOS PROFISSIONAL DE ENFERMAGEM DIANTE A MULHER COM O CÂNCER DE MAMA

Andressa Prates Sá

Dayane Araújo Rocha

Keilla Silva Santos

Ingrid Isabel de Andrade

Nhayeno Cordeiro Dantas

Vinícius Duarte Silva

Fernanda Santos Landim

Francielle Araujo Bispo

Weidny Eduardo de Sousa Silva

Cecília Rodrigues Lima

Anna Christina dos Reis Santos

Thais Pereira Silva

DOI: 10.47094/978-65-88958-64-3/37-46

CAPÍtULO 5.

A RELEVÂNCIA DA ASSISTÊNCIA DA EQUIPE DE ENFERMAGEM NA ORIENTAÇÃO DO ALEITAMENTO MATERNO

Andressa Prates Sá

Dayane Araújo Rocha

Keilla Silva Santos

Ingrid Isabel de Andrade

Nhayeno Cordeiro Dantas

Vinícius Duarte Silva

Fernanda Santos Landim

Francielle Araujo Bispo 
Weidny Eduardo de Sousa Silva

Cecília Rodrigues Lima

Anna Christina dos Reis Santos

Thais Pereira Silva

DOI: 10.47094/978-65-88958-64-3/47-57 


\section{CAPÍTULO 5}

\section{A RELEVÂNCIA DA ASSISTÊNCIA DA EQUIPE DE ENFERMAGEM NA ORIENTAÇÃO DO ALEITAMENTO MATERNO}

\section{Andressa Prates Sá}

Faculdade de Saúde e Humanidades Ibituruna - FASI, Montes Claros, Minas Gerais. https://orcid.org/0000-0002-9892-7191

\section{Dayane Araújo Rocha ${ }^{2}$}

Enfermeira Graduada Universidade do Estado de Minas Gerais.

https://orcid.org/0000-0001-9594-3287

\section{Keilla Silva Santos ${ }^{3}$}

Faculdade de Saúde e Humanidades Ibituruna- FASI, Montes Claros, Minas Gerais.

$\underline{\text { http://lattes.cnpq.br/4390347860194905 }}$

\section{Ingrid Isabel de Andrade ${ }^{4}$}

Faculdade de Saúde e Humanidades Ibituruna - FASI, Montes Claros, Minas Gerais. http://lattes.cnpq.br/8559852008026622

\section{Nhayeno Cordeiro Dantas ${ }^{5}$}

Faculdade de Saúde e Humanidades Ibituruna - FASI, Montes Claros, Minas Gerais.

\section{http://lattes.cnpq.br/5756148457135856}

\section{Vinícius Duarte Silva ${ }^{6}$}

Faculdade de Saúde e Humanidades Ibituruna - FASI, Montes Claros, Minas Gerais. http://lattes.cnpq.br/0383688388186075

\section{Fernanda Santos Landim ${ }^{7}$}

Graduada em Enfermagem pela Universidade Estadual de Montes Claros.

http://lattes.cnpq.br/8867397116277703

\section{Francielle Araujo Bispo ${ }^{8}$}

Faculdade de Saúde e Humanidades Ibituruna - FASI, Montes Claros, Minas Gerais. http://lattes.cnpq.br/8128766871065494 


\section{Weidny Eduardo de Sousa Silva9}

Faculdade de Saúde e Humanidades Ibituruna - FASI, Montes Claros, Minas Gerais.

http://lattes.cnpq.br/5249560920697190

\section{Cecília Rodrigues Lima ${ }^{10}$}

Faculdade de Saúde e Humanidades Ibituruna - FASI, Montes Claros, Minas Gerais. http://lattes.cnpq.br/1190180224007265

\section{Anna Christina dos Reis Santos ${ }^{11}$}

Faculdades Unidas do Norte de Minas - FUNORTE

http://lattes.cnpq.br/4145234426437540

\section{Thais Pereira Silva ${ }^{12}$}

Faculdade de Saúde e Humanidades Ibituruna- FASI, Montes Claros, Minas Gerais. $\underline{\text { http://lattes.cnpq.br/78744455243844985 }}$

RESUMO: Introdução: A prática da amamentação é um processo fisiológico, natural, constituindo a melhor forma de alimentar e preservar o recém-nascido. Dentre os benefícios do aleitamento materno para a mulher estão: mães que amamentam logo após o parto têm maior chance de serem bem sucedidas na prática da amamentação; os alimentos pré-lácteos, frequentemente oferecidos aos bebês antes da amamentação, podem causar lesões no intestino imaturo; o colostro acelera a maturação do epitélio intestinal e protege contra agentes patogênicos; o contato pele a pele previne a ocorrência de hipotermia. Objetivo: Identificar e descrever a relevância da assistência da equipe de enfermagem na orientação do aleitamento materno. Metodologia: Trata-se de um estudo de abordagem qualitativa, através da identificação de estudos sobre o tema e a relevância da assistência da equipe de enfermagem na orientação do aleitamento materno, entre os anos de 2008 e 2021. Adotou-se a revisão integrativa da literatura devido a sua contribuição para a análise de resultados. Resultados e Discussão: A assistência em enfermagem é primordial para o enfrentamento das dificuldades vivenciados pelas mães e pela família sendo que interferem na promoção do aleitamento materno, visto que enfermeiro(a) é considerado o profissional que mais se aproxima das mães, contudo tem uma função de suma importância nos programas de educação em saúde. O profissional deve possuir fundamento aproximadamente de várias informações, para planejar o cuidado com as famílias, com o objetivo de realizar um cuidado integral. Conclusão: Os benefícios do aleitamento materno estão claros para os profissionais de enfermagem como uma maneira de prevenir doenças e garantir à criança uma vida saudável e um desenvolvimento seguro. O enfermeiro deve ampliar sua participação na promoção ao aleitamento materno como estratégia eficiente de prevenção de adoecimento e otimização da qualidade de vida da população. 
PALAVRAS-CHAVE: Aleitamento Materno. Cuidados de Enfermagem. Amamentação.

ABSTRACT: Introduction: The practice of breastfeeding is a physiological, natural process, constituting the best way to feed and preserve the newborn. Among the benefits of breastfeeding for women are: mothers who breastfeed soon after birth are more likely to be successful in breastfeeding; pre-dairy foods, often given to babies before breastfeeding, can damage the immature bowel; colostrum accelerates the maturation of the intestinal epithelium and protects against pathogens; skinto-skin contact prevents hypothermia. Objective: To identify and describe the relevance of nursing staff assistance in breastfeeding guidance. Methodology: This is a study with a qualitative approach, through the identification of studies on the topic and the relevance of nursing staff assistance in guiding breastfeeding, between 2008 and 2021. The integrative review of the literature due to its contribution to the analysis of results. Results and discussion :Nursing care is essential for coping with the difficulties experienced by mothers and the family, interfering with the promotion of breastfeeding, as nurses are considered the professionals who are closest to mothers, however they have a role of paramount importance in health education programs. The professional must have a foundation approximately of various information, in order to plan care for the families, with the objective of providing comprehensive care. Conclusion: The benefits of breastfeeding are clear to nursing professionals as a way to prevent diseases and ensure a healthy life and safe development for the child. Nurses should expand their participation in promoting breastfeeding as an efficient strategy for preventing illness and optimizing the population's quality of life.

KEY-WORDS: Breastfeeding. Nursing care. Breast-feeding.

\section{INTRODUÇÃO}

A prática da amamentação é um processo fisiológico, natural, constituindo a melhor forma de alimentar e preservar o recém-nascido. O leite humano é formado de nutrientes em quantidade exata para o amadurecimento do cérebro humano, ao contrário do leite produzido por outros mamíferos e, muitas vezes, utilizado para alimentar o recém-nascido. O leite materno, além de demonstrar estabelecer perfeita afinidade nutricional, desempenha ação imunológica ao bebê (CUNHA; SIQUEIRA, 2016).

O Aleitamento Materno Exclusivo (AME) deve ser permanecido até o sexto mês de vida do bebê, de acordo recomendações do Ministério da Saúde (MS), Organização Mundial de Saúde (OMS) e Fundo das Nações Unidas para a Infância (UNICEF), devendo ser associado a outros alimentos dos seis meses completos até o segundo ano de vida (LUSTOSA; LIMA, 2020).

O sucesso do aleitamento materno se associa a programas educativos de diversas naturezas e ao reconhecimento da cultura especificamente relacionada a esta prática social. Além do mais, sua promoção e incentivo precisam ocorrer em todas as situações, para que as mães aprimorem seu conhecimento sobre o assunto e, consequentemente, aumentem sua prevalência e duração (OLIVEIRA 
et al., 2012).

Porém, a prática da amamentação sofre influência de vários aspectos: familiar, cultural, social, psíquico, biológico, espiritual, ambiental, entre outros, que tornam essa prática mais aceita, ou não, pela mãe. Além disso, existe a necessidade do estímulo e motivação dos profissionais de saúde qualificados para aumentar o conhecimento da mãe, incentivando-a para a prática do aleitamento materno (CUNHA; SIQUEIRA, 2016).

Dentre os benefícios do aleitamento materno para a mulher estão: mães que amamentam logo após o parto têm maior chance de serem bem sucedidas na prática da amamentação; os alimentos pré-lácteos, frequentemente oferecidos aos bebês antes da amamentação, podem causar lesões no intestino imaturo; o colostro acelera a maturação do epitélio intestinal e protege contra agentes patogênicos; o contato pele a pele previne a ocorrência de hipotermia (TOMA; REA; 2008).

Estudos recentes evidenciam que as diversas formas de ação e suas consequências para a saúde da criança mostraram que a promoção do aleitamento materno exclusivo é a intervenção isolada em saúde pública com o maior potencial para a diminuição da mortalidade na infância (TOMA; REA; 2008).

A Política Nacional de Atenção Integral à Saúde da Criança (PNAISC) é constituída por ações e estratégias que alvejam nortear a atenção à saúde quanto ao crescimento e desenvolvimento da criança no seu ciclo de vida, de acordo com as iniciativas e diretrizes do Sistema Único de Saúde (SUS). Baseiam-se na prevenção de agravos e doenças, promoção da saúde, assistência e reabilitação, respeitando os direitos à vida e à saúde da criança (SOUSA et al., 2019).

Neste contexto, ao enfermeiro, como integrante da equipe multiprofissional, cabe juntamente com os demais integrantes, a responsabilidade de ensinar, educar e assistir a população, visando à promoção, à proteção e à recuperação da saúde do indivíduo e da coletividade (CUNHA; SIQUEIRA, 2016).

O enfermeiro tem um papel essencial na educação e promoção da saúde na atenção primária, sendo o ator principal no quesito de ensinamento das gestantes durante todo o pré-natal, no puerpério e pós-parto até os 6 meses de vida do bebê. As vantagens de seguir o aleitamento materno exclusivo (AME) são incalculáveis, podendo ser destacados alguns aspectos fisiológicos importantes tanto para a mulher como para o bebê, sendo eles: involução uterina mais acelerada; diminuição das chances de uma nova gestação; recomposição corporal e prevenção de mastite puerperal; oferta de todos os nutrientes para um bom desenvolvimento; hidratação adequada e recebimento de células de defesa através do leite (LUSTOSA; LIMA, 2020).

No entanto, sucede a seguinte questão: as puérperas devem ter a assistência prestada pela equipe de enfermagem na primeira hora de vida do recém-nascido quanto ao incentivo ao aleitamento materno (LEITE, et al., 2016).

A criação de estratégias para direcionar as mães que, por preferência ou obrigação, decidiram amamentar ou não, permanece presente nas ações de saúde. O profissional da saúde é uma ferramenta 
fundamental para promoção, proteção e ajuda na amamentação, por realizar estratégias diretas que vão beneficiar a mãe e o filho, por meio de ações educativas, de técnicas de amamentação, apoio emocional, verbal e na criação de grupos que vai reunir gestantes para troca de informações (LEITE, et al., 2016).

Este mesmo profissional deve dispor de uma visão integral a fim de identificar na gestante os seus conhecimentos, suas experiências práticas, suas crenças e valores, além da vivência social e familiar com a finalidade de promover a educação em saúde para o Aleitamento Materno Exclusivo $(\mathrm{AME})$ e assim garantir assistência e efetividade durante a assistência à nutriz no pós-parto imediato (LUSTOSA; LIMA, 2020).

O profissional de enfermagem exerce um papel fundamental no que se refere ao aconselhamento das futuras mamães, sendo um meio importante para aumentar o índice das mães que amamentam, este deve apoiar e orientar a gestante durante o pré-natal, formando grupos de gestantes e promovendo campanhas de incentivo ao aleitamento (MESQUITA, et al., 2016).

O profissional de enfermagem deve ser capacitado para melhor informar e tirar dúvidas das gestantes, o que se inicia no pré-natal discutindo sobre a importância do aleitamento com as mães. Diante de uma realidade onde há necessidade de mudanças, faz-se necessário fortalecer a atuação do enfermeiro em benefício do aleitamento materno, de forma a aumentar o número de mães que aderem a esta prática que é de extrema importância para o desenvolvimento do bebê; desta forma este estudo terá como objetivo identificar as atribuições do enfermeiro no processo de conscientização do aleitamento materno para com as gestantes (MESQUITA, et al., 2016).

\section{OBJETIVO}

Com base nessas considerações, o presente estudo tem como objetivo revisar a literatura científica com a finalidade de identificar e descrever a relevância da assistência da equipe de enfermagem na orientação do aleitamento materno.

\section{METODOLOGIA}

Trata-se de um estudo de abordagem qualitativa, através da identificação de estudos sobre o tema e a relevância da assistência da equipe de enfermagem na orientação do aleitamento materno, entre os anos de 2008 e 2021. Adotou-se a revisão integrativa da literatura devido a sua contribuição para a análise de resultados.

A revisão integrativa da literatura estabelece critérios para a coleta e análise de dados, desta forma adotamos seis etapas indicadas para a elaboração da revisão integrativa que são: a seleção da pergunta em que consiste a pesquisa; a definição dos critérios de inclusão dos estudos e seleção da amostra; a representação dos estudos selecionados em tabela, representado na tabela 1; análise crítica dos dados encontrados e a discussão dos resultados obtidos. 
A seleção dos estudos foi realizada através de busca em bancos de dados online como: Scielo, google acadêmico, BMV, Pubmed e MedLine, com base nos critérios: ano de publicação entre 2008 e 2021; publicados em português, espanhol e inglês e artigos com textos completos. Foram utilizados 10 artigos, e referenciados no presente texto descritos na tabela 1.

\section{RESULTADOS E DISCUSSÃO}

Tabela 1 - Descrição de trabalhos selecionados para a revisão integrativa.

\begin{tabular}{|c|c|c|}
\hline AUTOR/ ANO & TÍTULO & OBJETIVO \\
\hline $\begin{array}{l}\text { CARVALHO; CARVALHO; MA- } \\
\text { GALHÃES, } 2011 .\end{array}$ & $\begin{array}{l}\text { A importância da assistência } \\
\text { de enfermagem no aleitamento } \\
\text { materno. }\end{array}$ & $\begin{array}{l}\text { Deste trabalho é descrever através } \\
\text { de um estudo exploratório embasado } \\
\text { em levantamento teórico científico } \\
\text { a importância da assistência de en- } \\
\text { fermagem para o alcance do sucesso } \\
\text { no aleitamento materno, bem como } \\
\text { a necessidade e importância da ama- } \\
\text { mentação com orientações básicas a } \\
\text { puérpera e familiares. }\end{array}$ \\
\hline CUNHA; SIQUEIRA, 2016. & $\begin{array}{l}\text { Aleitamento Materno: Contri- } \\
\text { buições da Enfermagem }\end{array}$ & $\begin{array}{l}\text { Conhecer a produção científica sobre } \\
\text { aleitamento materno e as contribui- } \\
\text { ções de enfermagem nessa prática. }\end{array}$ \\
\hline SOUSA et al., 2019. & $\begin{array}{l}\text { Desafios e potencialidades na } \\
\text { assistência de enfermagem no } \\
\text { aleitamento materno. }\end{array}$ & $\begin{array}{l}\text { Caracterizar as principais dificulda- } \\
\text { des e potencialidades da assistência } \\
\text { de enfermagem durante o período de } \\
\text { aleitamento materno. }\end{array}$ \\
\hline FERREIRA, et al., 2016. & $\begin{array}{l}\text { O Papel da Enfermagem na } \\
\text { orientação do aleitamento ma- } \\
\text { terno exclusivo. }\end{array}$ & $\begin{array}{l}\text { Retratar a importância da orientação } \\
\text { da enfermagem no aleitamento ma- } \\
\text { terno. }\end{array}$ \\
\hline LEITE, et al., 2016. & $\begin{array}{l}\text { Promoção do aleitamento ma- } \\
\text { terno na primeira hora de vida } \\
\text { do recém-nascido por profissio- } \\
\text { nais da enfermagem. }\end{array}$ & $\begin{array}{l}\text { O descrever e analisar a percepção } \\
\text { das puérperas acerca do incentivo ao } \\
\text { aleitamento materno na primeira hora } \\
\text { de vida pelos profissionais de enfer- } \\
\text { magem em uma maternidade pública. }\end{array}$ \\
\hline LUSTOSA; LIMA, 2020. & $\begin{array}{c}\text { Importância da enfermagem } \\
\text { frente à assistência primária ao } \\
\text { aleitamento materno exclusivo } \\
\text { na atenção básica. }\end{array}$ & $\begin{array}{l}\text { Importância de verificar a prática dos } \\
\text { profissionais de enfermagem rela- } \\
\text { cionada ao aleitamento materno, no } \\
\text { período gravídico e puerperal especi- } \\
\text { ficamente. }\end{array}$ \\
\hline MESQUITA, et al., 2016. & $\begin{array}{l}\text { Atribuições de enfermeiros na } \\
\text { orientação de lactantes acerca } \\
\text { do aleitamento materno. }\end{array}$ & $\begin{array}{l}\text { Identificar as atribuições do enfer- } \\
\text { meiro no processo de conscientização } \\
\text { do aleitamento materno para com as } \\
\text { gestantes. }\end{array}$ \\
\hline
\end{tabular}




\begin{tabular}{|c|c|c|}
\hline OLIVEIRA, et al., 2012. & $\begin{array}{c}\text { Aleitamento materno: conheci- } \\
\text { mento e prática. }\end{array}$ & $\begin{array}{c}\text { Caracterizar as práticas de promoção } \\
\text { ao aleitamento materno desenvol- } \\
\text { vidas pelos profissionais de enfer- } \\
\text { magem da Estratégia de Saúde da } \\
\text { Família e analisar a correlação entre } \\
\text { seu conhecimento sobre aleitamen- } \\
\text { to materno e a frequência com que } \\
\text { realizavam orientações sobre o tema } \\
\text { nesses momentos. }\end{array}$ \\
\hline PALHETA; REA; 2008. & $\begin{array}{c}\text { Benefícios da amamentação } \\
\text { para a saúde da mulher e da } \\
\text { criança: um ensaio sobre as } \\
\text { evidências }\end{array}$ & $\begin{array}{c}\text { Avaliar quais intervenções seriam } \\
\text { mais efetivas para um aumento das } \\
\text { práticas de amamentação. }\end{array}$ \\
\hline $\begin{array}{c}\text { Importância da assistência de } \\
\text { enfermagem para a promoção } \\
\text { do }\end{array}$ & $\begin{array}{c}\text { O objetivo geral deste artigo é } \\
\text { dissertar sobre a importância da } \\
\text { assistência em enfermagem para } \\
\text { estimular a promoção ao aleitamento } \\
\text { materno }\end{array}$ \\
\hline
\end{tabular}

Fonte: próprios autores. Uma revisão integrativa da literatura.

Segundo os estudos de Mesquita et al., (2016) o enfermeiro deve ser devidamente capacitado, ter conhecimento, habilidades e sensibilidade para aconselhamento, compreendendo a amamentação como um processo complexo que engloba a cultura, o valor, o social, o biológico e o emocional, indo além das informações técnicas ampliando a assistência associada a aspectos socioculturais.

De acordo com Palheta e Aguiar (2021) sabendo que existem dificuldades na realização do aleitamento materno, principalmente nos primeiros dias de vida, o profissional de enfermagem atribui um essencial papel perante a promoção desta prática. $\mathrm{O}$ enfermeiro deve representar como profissional educador na intenção de aumentar o interesse pelo estilo de vida saudável, realizar educação prolongada para a promoção do aleitamento materno.

Diante dos estudos de Mesquita et al., (2016) o enfermeiro realiza consultas de enfermagem em domicílio, às gestantes que não encontram as medidas sociais, usualmente são mulheres de menor situação econômica, solteiras e que trabalham, para um acompanhamento individual, as orientações sobre a importância do aleitamento materno têm que ser ofertadas tanto a parturiente em que ao recém-nascido.

De acordo com Palheta e Aguiar (2021) a assistência em enfermagem é primordial para o enfrentamento das dificuldades vivenciados pelas mães e pela família sendo que interferem na promoção do aleitamento materno, visto que enfermeiro(a) é considerado o profissional que mais se aproxima das mães, contudo tem uma função de suma importância nos programas de educação em saúde. e relevante a esses profissionais a incentivar e encorajar a mãe a praticar o aleitamento materno 
a partir da compreensão sobre o seu contexto sociocultural e familiar.

De acordo com Lustosa e Lima (2020) em um determinado estudo sobre enfermagem e AME, o autor aponta que somente o fornecimento de conhecimentos ou práticas educativas em saúde são estratégias ineficazes para influenciar as mães quanto ao ato de amamentar sendo essencial que sejam ofertadas medidas concretas para que o binômio tenha esse processo de forma prazerosa e com eficiência. Todavia, o enfermeiro deve proporcionar a expansão de sua área de atuação, superando as questões fisiológicas da amamentação, conquistando a confiança da mulher e se tornando uma ferramenta que transmite conhecimento a ela, estando apto a suprir suas percepções acerca do AME e propulsionando a compreensão sobre a amamentação, realizando uma assistência holística e integral.

Segundo Oliveira et al., (2012) a partir da constatação de que o desmame precoce incluir num contexto social, educacional e de responsabilidade dos serviços de saúde, destaca a necessidade de desenvolvimento de medidas para a amamentação, com vistas a sustentar a prática do aleitamento materno por seis meses.

Segundo os estudo de Mesquita et al., (2016) o desempenho do enfermeiro é de prevenção e promoção à saúde da gestante por meio de projetos e medidas sociais que são promovidas nos postos de Estratégia Saúde da Família contém acompanhada normas da OMS, efetuada exames como teste da mãezinha gratuito realizado pelo SUS que abrange enfermidades têm como exemplo: Citomegalovirus, Clamídia, Doença de Chagas, Hepatite B, Hiperfenilalaninemia Materna, Hipotireoidismo Materno, HIV, Rubéola, Sífilis, Toxoplasmose e Variantes de Hemoglobinas.

Segundo os estudos obtidos por Carvalho, Carvalho e Magalhães (2011), o aleitamento materno é uma das maneiras mais eficientes de atender os pontos de vista nutricionais, imunológicos e psicológicos da criança em seu primeiro ano de vida, tornando se uma prática natural e efetivo, que propiciar o vínculo mãe-filho em que momento o ato de amamentar é bem vivenciado pelas mães. É um ato cujo circunstanciado de fatores históricos, sociais, culturais, e psicológicos da puérpera, depende de compromisso e entendimento técnico-científico dos profissionais de saúde compreendidos na promoção, incentivo e auxiliando ao aleitamento materno.

De acordo com Lustosa e Lima (2020), a assistência de enfermagem deve ser prestada com orientações em como realizar a amamentação com técnica adequada, posição e pegada correta; deve prestar conhecimento com relação aos cuidados que devem ser tomados com os mamilos para mantê-los secos, orientar a necessidade de fazer exposição ao ar livre ou luz solar e realizar trocas frequentes dos forros usados em que momento ocorre o vazamento de leite; ter o cuidado para não usar produtos que retirem a proteção natural do mamilo, como álcool, sabão ou qualquer produto secante; não ter restrições ao colocar a criança para mamar.

Além de ser efetivar as explicações de como evitar ingurgitamento mamário e de como aumentar a flexibilidade da aréola através da ordenha manual antes de colocar a bebê para mamar, caso esta identificar-se ingurgitada, bem como auxiliando para pegada adequada, ser esclarecido que não use protetores de mamilos, pois eles, além de não serem eficazes podem causar ou serem responsáveis pelo trauma mamilar (LUSTOSA; LIMA,2020). 
De acordo com Carvalho, Carvalho e Magalhães (2011), para que o enfermeiro coordene as suas tarefas, desde a administração até as atividades assistenciais, é essencial que ele sistematize a sua assistência para favorecer a solução das adversidades, agilizar e dinamizar suas medidas. Assim as perspectivas do enfermeiro obtiveram organização e sequência em suas atividades, evitando lacunas na assistência.

Dada à importância da atuação do profissional de enfermagem frente à amamentação, dado que o enfermeiro é o profissional que mais estreita relação com a mulher no decorrer o ciclo gravídico-puerperal e essencial no papel nos programas de educação em saúde e durante o pré-natal, este profissional tem a importante função de preparar a gestante para o aleitamento e para o pós-parto, para que a puérpera se adapte da melhor maneira ao aleitamento, evitando assim, dúvidas, dificuldades e possíveis complicações (FERREIRA, et al., 2016).

Carvalho, Carvalho e Magalhães (2011), mesmo que os profissionais de saúde procuram desempenhar medidas específicas dentro de sua formação acadêmica no decorrer da a assistência de promoção, incentivo e apoio ao aleitamento materno, políticas institucionais devem assegurar o exercício profissional de todos e apoiar a diversificação de suas atuações em vantagens da mulher e da criança. Estudar a ação do enfermeiro na assistência ao aleitamento materno ao longo da hospitalização da puérpera é uma forma de destacar o seu papel e a importância de sua atuação, bem como da sistematização da assistência de enfermagem.

De acordo com Carvalho, Carvalho e Magalhães (2011), é primordial que o enfermeiro saiba a relevância da amamentação e os benefícios que este alimento traz para a vida da criança e da mãe. $\mathrm{O}$ profissional deve possuir fundamento aproximadamente de várias informações, para planejar o cuidado com as famílias, com o objetivo de realizar um cuidado integral.

Segundo os estudos de Carvalho, Carvalho e Magalhães (2011), o papel do Enfermeiro consiste em orientar a mulher e seu companheiro acerca das vantagens da amamentação, para a criança, para a família, principalmente para a proporcionar mulher que amamenta. reconhecer leituras e materiais educativos aos pais, que tendem de disposição nos serviços de pré-natal. No decorrer dos encontros, a enfermeira deve instigar a mulher a fazer perguntas, a explicar acerca de prováveis dúvidas, tabus habituais na comunidade, e disponibilizar as informações adicionais.

De acordo com Carvalho, Carvalho e Magalhães (2011), o ensinamento sobre aleitamento materno é de substancial importância, onde o enfermeiro tem a oportunidade de acontecer não somente atividades educativas, além do mais a assistenciais, principalmente nas patologias comuns durante o início da amamentação, responsáveis algumas vezes, até mesmo pelo desmame precoce.

\section{CONCLUSÃO}

Diante desse estudo, o papel da enfermagem é garantir por meio da promoção, proteção e prevenção a prática do aleitamento materno, não só através da informação, mas principalmente pela implementação de medidas que envolvam a gestante e sua família durante o pré-natal, parto e pós- 
-parto, contribuindo assim como para a correta condição de aleitamento materno.

No entanto, a amamentação é essencial para a saúde do bebê, e também para a saúde e recuperação pós-parto da mãe. Os benefícios da amamentação para a saúde da mulher precisam ser mais estudados. $\mathrm{O}$ aleitamento proporciona também à criança inúmeros benefícios, dentre os quais crescimento infantil adequado, proteção contra infecções, melhor desenvolvimento da musculatura da cavidade bucal, com efeitos positivos inclusive na inteligência, e, ainda, diminuição do risco de alergias, hipertensão arterial, hipercolesterolemia e diabetes mellitus, limitando a chance de obesidade, entre outros.

Os benefícios do aleitamento materno estão claros para os profissionais de enfermagem como uma maneira de prevenir doenças e garantir à criança uma vida saudável e um desenvolvimento seguro. O enfermeiro deve ampliar sua participação na promoção ao aleitamento materno como estratégia eficiente de prevenção de adoecimento e otimização da qualidade de vida da população.

\section{DECLARAÇÃO DE INTERESSES}

Nós, autores deste capítulo, declaramos que não possuímos conflitos de interesses de ordem financeira, comercial, política, acadêmica e pessoal.

\section{REFERÊNCIAS}

DE CARVALHO, Janaina Keren Martins; CARVALHO, Clecilene Gomes; MAGALHÃES, Sérgio Ricardo. A importância da assistência de enfermagem no aleitamento materno. E-scientia, v. 4, n. 2, p. 11-20, 2011.

DA CUNHA, Élida Caetano; DE SIQUEIRA, Hedi Crecencia Heckler. Aleitamento materno: contribuições da enfermagem. Ensaios e Ciência: Ciências Biológicas, Agrárias e da Saúde, v. 20, n. 2 , p. 86-92, 2016.

LUSTOSA, Evaldo; LIMA, Ronaldo Nunes. Importância da enfermagem frente à assistência primária ao aleitamento materno exclusivo na atenção básica. Revista Brasileira Interdisciplinar de Saúde, 2020.

FERREIRA, Gabriela Rodrigues et al. O papel da enfermagem na orientação do aleitamento materno exclusivo. Revista Conexão Eletrônica, v. 13, n. 1, p. 1-18, 2016.

DA SILVA LEITE, Maura Fernanda Ferreira et al. Promoção do aleitamento materno na primeira hora de vida do recém-nascido por profissionais da enfermagem. Arquivos de Ciências da Saúde da UNIPAR, v. 20, n. 2, 2016.

MESQUITA, Ariele Londres et al. Atribuições de enfermeiros na orientação de lactantes acerca do aleitamento materno. Revista de Divulgação Científica Sena Aires, v. 5, n. 2, p. 158-170, 2016. 
FONSECA-MACHADO, Mariana de Oliveira et al. Aleitamento materno: conhecimento e prática. Revista da Escola de Enfermagem da USP, v. 46, p. 809-815, 2012.

TOMA, Tereza Setsuko; REA, Marina Ferreira. Benefícios da amamentação para a saúde da mulher e da criança: um ensaio sobre as evidências. Cadernos de Saúde Pública, v. 24, p. s235-s246, 2008. Palheta, Q. A. F., \& Aguiar, M. D. F. R. (2021). Importância da assistência de enfermagem para a promoção do aleitamento materno. Revista Eletrônica Acervo Enfermagem, 8, e5926-e5926. 


\section{ÍNDICE REMISSIVO}

$\mathbf{A}$

ação da enfermagem no contexto domiciliar 13, 18

agentes patogênicos 48,50

aleitamento materno $48,49,50,51,52,53,54,55,56,57$

alimentos pré-lácteos 48,50

alterações metabólicas 29, 30

amamentação $48,49,50,51,52,53,54,55,56,57$

assistência de enfermagem 13, 15, 16, 17, 18, 19, 24, 26, 29, 32, 38, 43, 44, 45, 46, 52, 53, 54, 55, 56,57

assistência de enfermagem aos portadores de Hipertensão Arterial Sistêmica 29

atividades de autocuidado 13,18

atuação da enfermagem na prevenção, diagnóstico e tratamento da Hanseníase 13, 15

B

bacilo de Hansen 13, 14

benefícios do aleitamento materno 48

C

câncer $21,22,23,24,25,26,33,38,39,40,41,42,43,44,45,46$

câncer cérvico uterino 21,22

câncer de colo de útero 21, 23, 24, 25, 26

carcinoma de útero 21,25

colostro 48,50

D

deserção do tabagismo 29, 31

dificuldade da hanseníase 13

dificuldades vivenciados pelas mães 48,53

doença circulatória 29, 30

doença infectocontagiosa crônica 13, 14

doenças cardiovasculares fatais e não fatais 29,30

$\mathbf{E}$

educação dos portadores de hanseníase 13

educação em saúde 13, 17, 18, 24, 26, 33, 34, 36, 48, 51, 53, 55

equipe de enfermagem na assistência do câncer de colo do útero 21, 23, 24

equipe de enfermagem na orientação do aleitamento materno 48 
exame citopatológico 21, 23

exercícios físicos $25,29,31,34$

G

gestantes $21,23,50,51,52,53$

H

hábitos de vida 29, 31, 33, 38, 40

hanseníase 13, 14, 15, 16, 17, 18, 19

Hipertensão Arterial 29, 30, 31, 32, 35

hipotermia 48, 50

histerectomia parcial 21, 23

I

incapacidades físicas 13,17

$\mathbf{L}$

lesões no intestino imaturo 48, 50

M

mamografia 38, 40, 44

maturação do epitélio intestinal 48,50

menopausa 21, 23

mulheres com câncer de mama 38, 41

Mycobacterium leprae 13, 14

$\mathbf{N}$

neoplasia $38,39,44$

neoplasia mamária 38, 44

$\mathbf{P}$

parasita intracelular obrigatório 13, 14

perda do controle da divisão celular 21,22

portadores de Hanseníase 13, 15

prática da amamentação 48

processo de adoecimento 38,41

Q

qualidade de vida $16,25,31,34,35,48,56$

$\mathbf{R}$

recém-nascido $48,49,50,52,53,56$ 
saúde pública 29, 34, 38, 39, 44, 50

T

tratamento do câncer de colo de útero 21, 25

V

vida sexual 21,23

vida sexual ativa 21,23

virgens 21,23 
editoraomnisscientia@gmail.com M https://editoraomnisscientia.com.br/ $\circledast$

@editora_omnis_scientia (0) https://www.facebook.com/omnis.scientia.9 f

$$
\text { +55 (87) 9656-3565 }
$$


editoraomnisscientia@gmail.com M https://editoraomnisscientia.com.br/ $\circledast$

@editora_omnis_scientia (0) https://www.facebook.com/omnis.scientia.9 f

$$
\text { +55 (87) 9656-3565 }
$$

